\title{
Short-Term Arterial Compliance Changes in the Context of Systolic Blood Pressure Influence
}

\author{
David SVEC ${ }^{1}$, Barbora CZIPPELOVA ${ }^{1,2}$, Jana CERNANOVA KROHOVA ${ }^{1,2}$, Nikoleta \\ MAZGUTOVA ${ }^{1}$, Radovan WISZT ${ }^{1}$, Zuzana TURIANIKOVA ${ }^{1,2}$, Lenka MATUSKOVA ${ }^{1}$, \\ Michal JAVORKA ${ }^{1}$
}

${ }^{1}$ Department of Physiology, Comenius University in Bratislava, Jessenius Faculty of Medicine in Martin, Martin, Slovak Republic, ${ }^{2}$ Biomedical Centre Martin, Comenius University in Bratislava, Jessenius Faculty of Medicine in Martin, Martin, Slovak Republic

Received April 15, 2021

Accepted November 11, 2021

\section{Summary}

Arterial compliance (C) is a complex parameter influencing ventricular-arterial coupling depending on structural (arterial wall remodeling) and functional (blood pressure, smooth muscles tone) changes. Based on Windkessel model, $\mathrm{C}$ can be calculated as the ratio of a time constant Tau characterizing diastolic blood pressure decay and total peripheral resistance (TPR). The aim of this study was to assess changes of $C$ in the context of systolic arterial pressure (SAP) perturbations during four physiological states (supine rest, head-up tilt, supine recovery, mental arithmetic). In order to compare pressure independent changes of $C$ a new index of $C_{120}$ was proposed predicting $C$ value at $120 \mathrm{~mm} \mathrm{Hg}$ of SAP. Eighty-one healthy young subjects (48 f, average age 18.6 years) were examined. Hemodynamic parameters were measured beat-to-beat using volume-clamp photoplethysmographic method and impedance cardiography. We observed that $C$ was strongly related to SAP values on the beat-to-beat time scale. Interestingly, $\mathrm{C}_{120}$ decreased significantly during stress phases. In conclusion, potential changes of SAP should be considered when measuring $C$. Arterial compliance changes in the opposite direction to TPR pointing towards influence of vascular tone changes on its value.

\section{Key words}

Arterial compliance - Systolic pressure - Total peripheral resistance $\bullet$ Head-up tilt $\bullet$ Mental stress

\section{Corresponding author}

D. Svec, Department of Physiology Jessenius Faculty of Medicine in Martin, Comenius University in Bratislava, Mala Hora 4C, 03601 Martin, Slovak Republic. E-mail: svec70@uniba.sk

\section{Introduction}

Arterial compliance (C) is an important index characterizing mechanical vascular properties significantly influencing ventricular-arterial coupling. Decreased $\mathrm{C}$ is associated with various physiological (age (McEniery et al. 2005, O'Rourke and Hashimoto 2007)) and pathophysiological (hypertension, diabetes, atherosclerosis (Chemla et al. 2003, Nagai et al. 2001)) processes and thus its estimation has for a long time interested clinicians and cardiovascular physiologists. However, the noninvasive estimation of the $\mathrm{C}$ is still a challenging task. In order to estimate the value of $\mathrm{C}$ or its reciprocal value - arterial stiffness, numerous methods or surrogate indexes were proposed (Hallock and Benson 1937, Levenson et al. 1981, Stergiopulos et al. 1995, Vlachopoulos et al. 2006). Currently, pulse wave velocity (PWV) is considered as the gold standard of non-invasive and reproducible method to determine arterial stiffness (Laurent et al. 2006).

Besides PWV, several methods for estimation of $\mathrm{C}$ were developed based on the Windkessel model of cardiovascular system. In this model, cardiovascular system is simplified into a set of tubes with two or more parameters, depending on the model complexity (Sagawa et al. 1990). The simplest Windkessel model is described by two parameters - $\mathrm{C}$ and total peripheral resistance (TPR). According to this model, time constant $\tau$ - the rate of the arterial blood pressure decay during diastolic phase - is the product of these two parameters. Given the relationships between $\mathrm{C}$, TPR and $\tau, \mathrm{C}$ value can be 
calculated as the ratio of $\tau$ over TPR. However, a determination of $\tau$ is associated with methodological difficulties, i.e. arterial blood pressure curve has to be measured centrally - e.g. in the aortic arch - because of the distortions in peripheral arterial blood pressure curve due to pulse wave reflections (Kroeker and Wood 1955). Today, the most reliable method of central blood pressure curve recording is using catheter equipped with a pressure sensor. This solution is invasive, therefore, its usability is limited to the patients with intra-aortic measurement of blood pressure.

Furthermore, when interpreting changes in $\mathrm{C}$ as an index of structural arterial changes (mostly related to atherosclerotic process), several confounders potentially influencing estimated value should be considered, including arterial pressure (Cohen et al. 2020, Hallock and Benson 1937), smooth muscle cells tone (Bank et al. 1995, Cox 1975, Czippelova et al. 2019) related to sympathetic nerve activity (Nardone et al. 2018), and heart rate (Lantelme et al. 2002, Tan et al. 2016). A better understanding of changes in $\mathrm{C}$ related to above mentioned confounding factors could improve our understanding of short-term and long-term $\mathrm{C}$ variability. It is important to take into consideration potential shortterm $\mathrm{C}$ changes when interpreting $\mathrm{C}$ value as an index of clinically important vascular stiffening.

Therefore, the aim of this study was to develop the methodology for the noninvasive beat-to-beat estimation of $\mathrm{C}$ and to assess pressure-independent changes of $\mathrm{C}$ in the context of alterations in vasomotor tone (manifested by changes in TPR) during standardized protocol including resting phases, orthostatic stress and cognitive load. To solve this task, we used recently developed method for continuous and non-invasive determination of $\tau$ using only parameters which are known to be robust against distortions due to wave reflections. It makes this method applicable on the peripheral blood pressure curve obtained by volumeclamp photoplethysmographic method (Arai et al. 2011). Together with calculated TPR values from the ratio of mean arterial pressure (MAP) and cardiac output (CO) obtained using impedance cardiography we calculated $\mathrm{C}$ values as the ratio of $\tau$ and TPR for each heart beat.

\section{Methods}

\section{Subjects}

In this study a total of 81 young and healthy Caucasians (48 females), aged 18.56 years
( $\mathrm{SD}=2.88$ years), were enrolled. All subjects were normotensive, nonobese $\left(\mathrm{BMI}=21.74 \mathrm{~kg} / \mathrm{m}^{2}\right.$, $\mathrm{SD}=2.32 \mathrm{~kg} / \mathrm{m}^{2}$ ) and were not taking any medications at the time of the study. Subjects were instructed not to use substances influencing autonomic nervous system or the cardiovascular system activity during $24 \mathrm{~h}$ before the examination. All measurements took place in the morning hours (from 8 am to $11 \mathrm{am}$ ) in a quiet examination room with ambient temperature ranging between 22 and $25^{\circ} \mathrm{C}$. Female subjects were examined during the proliferative phase $\left(5^{\text {th }}-12^{\text {th }}\right.$ day) of their menstrual cycle. All subjects or their legal representatives (in participants under 18 years of age) gave written informed consent prior to the examination. The study was approved by the ethical committee of the Jessenius Faculty of Medicine, Comenius University.

\section{Study protocol}

Subjects were positioned on a motorized tilt table with a foot support. A restraining strap secured at the thigh level was used to provide additional support and safety. Subjects were asked to avoid unnecessary speaking and moving during the measurement. The study protocol consisted of four consecutive phases:

1. supine rest (REST, $15 \mathrm{~min}$ ),

2. head-up tilt (HUT, the subject was tilted to 45 degrees for $8 \mathrm{~min}$ to evoke mild orthostatic stress),

3. supine recovery (REC, recovery from HUT for $10 \mathrm{~min})$,

4. mental arithmetic (MA, mental arithmetic task in the supine position for $6 \mathrm{~min}$ to evoke mental stress).

During the non-verbal MA task, subjects were instructed to sum up digits of randomly generated threedigit number until a one-digit number was reached and to decide whether the final result is odd or even. After that, the subject had to click on the corresponding button (indicated as "odd" or "even") projected on the ceiling with a wireless mouse. The sequence of three-digit numbers was projected on the ceiling of the examination room. During the MA task, rhythmic sound was generated by the metronome to disturb the examined subject. Subjects were instructed to perform the calculations without verbalization as quickly and as accurately as possible.

\section{Data acquisition}

We simultaneously and noninvasively recorded 
arterial blood pressure curve from finger with the subsequent brachial arterial blood pressure reconstruction by the photoplethysmographic volume-clamp method (Finometer Pro, FMS Netherlands). A hydrostatic height correction unit was attached on the measured arm at the level of aorta to correct for effects of hydrostatic pressure associated with the vertical distance between the aorta and finger. RR intervals were recorded by ECG (horizontal bipolar lead; CardioFax ECG-9620, NihonKohden Japan). In addition, hemodynamics parameters including stroke volume (SV) and CO were recorded by the impedance cardiography (CardioScreen 2000, Medis, Germany). All signals were digitized at a sampling rate of $1 \mathrm{kHz}$.

\section{Data extraction}

To exclude transient changes in cardiovascular parameters between consecutive phases of the study protocol from analysis, we extracted continuous segments of 300 beats from the original recordings as follows:

1. REST phase - started 8 min after the beginning of the measurement,

2. HUT phase - started $3 \mathrm{~min}$ after the change of position from supine to tilt,

3. REC phase - started 3 min after the subject was tilted back to supine position,

4. MA phase - started 2 min after the beginning of the calculation task.

Measurement of hemodynamic parameters using impedance cardiography is very sensitive to movement artifacts, skin condition and distribution of fat. Sometimes, we were not able to determine hemodynamic parameters for each heart beat because of these limitations. Only subjects with all four segments containing at least 250 of artifact free heart beats were included for further analysis.

\section{Determination of arterial compliance}

In order to quantify the value of $\mathrm{C}$, we applied a recently developed method (Arai et al. 2011). This approach allows to estimate time constant $\tau$ for each heart beat separately $\left(\tau_{i}\right)$. Estimation of $\tau_{i}$ is based only on parameters which are not influenced by distortion due to pulse wave reflection - cardiac cycle duration $\left(\mathrm{T}_{\mathrm{i}}\right)$ measured between present diastolic arterial pressure value $\mathrm{DAP}_{\mathrm{i}}$ and preceding $\mathrm{DAP}$ value $\left(\mathrm{DAP}_{\mathrm{i}-1}\right)$, mean arterial pressure during this cardiac cycle $\left(\mathrm{MAP}_{\mathrm{i}}\right)$ and time interval from the onset of systolic increase of blood pressure (at the moment of $\mathrm{DAP}_{\mathrm{i}-1}$ occurrence) to the following systolic arterial pressure peak $\left(\mathrm{Ts}_{\mathrm{i}}\right)$.

The advantage of this method is that time constant $\tau$ is calculated exclusively by the parameters, which are obtainable from the peripheral arterial blood pressure curve.

Furthermore, based on the Windkessel model, time constant $\tau$ is a product of C and TPR:

$$
\tau=C \cdot T P R
$$

While TPR is given by:

$$
T P R=\frac{M A P}{C O}
$$

Therefore, $\mathrm{C}$ can be calculated according to the following equation:

$$
C=\frac{\tau}{T P R}
$$

Monitoring relationship between arterial compliance and pressure in the context of vasomotor tone alterations

$\mathrm{C}$ is strongly dependent on arterial blood pressure making interpretation of its changes during perpetual arterial blood pressure perturbations very complicated. Therefore, we propose a new simple index $\mathrm{C}_{120}$ - for the quantification of arterial pressure independent changes in $\mathrm{C}$. This index is derived from the relationship between $\mathrm{C}$ and arterial pressure which is by nature nonlinear (Bank et al. 1995, Gavish and Izzo 2016). As a next step, we fit an exponential function to measured beat-to-beat data relating $\mathrm{C}$ to SAP for each phase separately:

$$
\mathrm{C}=\mathrm{a} \cdot \mathrm{e}^{-\mathrm{SAP} \cdot \mathrm{b}}+\mathrm{c}
$$

where $\mathrm{a}, \mathrm{b}$ and $\mathrm{c}$ are parameters to be estimated by fitting measured $\mathrm{C}$ and SAP values.

We decided to use SAP as a pressure parameter because of its largest variability within subject during study protocol. In addition, SAP is the pressure causing the most prominent stretching of elastic arteries an effect significantly influencing their compliance. Finally, using the fitted function, we predicted the value of $\mathrm{C}$ at $120 \mathrm{~mm} \mathrm{Hg}$ of SAP. We chose to use the value of SAP at $120 \mathrm{~mm} \mathrm{Hg}$ as a reference value typical for healthy individuals.

Furthermore, changes of $\mathrm{C}$ were interpreted in the context of alterations of vascular resistance (TPR) reflecting changes in sympathetic activity directed to the 
vessels to elucidate possible mechanism of observed differences.

\section{Statistics}

The Shapiro-Wilk test was used to determine the normality of all assessed variables. Paired $t$-test was used to compare parameters between baseline (REST, REC) and stress phases (HUT, MA), however, if violations of normality occurred, the Wilcoxon signed rank test was applied. A $p<0.05$ was considered statistically significant.

\section{Results}

\section{Changes of basic cardiovascular parameters}

Measured parameters are presented in Table 1. Each variable significantly changed from preceding baseline phases (REST, REC) to corresponding stress phase (HUT, MA). HUT induced an increase in heart rate (HR) and DAP, whereas SAP, MAP and PP decreased. In response to $\mathrm{MA}$, an increase was observed in each parameter. Throughout the whole study, the changes in SAP were more prominent than changes in MAP or DAP.

Table 1. Changes of basic cardiovascular parameters between baseline and stress phases.

\begin{tabular}{lccccccc}
\hline Variable & Rest & HUT & p & REC & MA & p & Distribution \\
\hline HR (beats $/ \mathrm{min})$ & $66.9(6.4)$ & $84.6(7.6)$ & $<0.001$ & $65.3(6.7)$ & $76.4(6.8)$ & $<0.001$ & non-Gaussian \\
SAP $(\mathrm{mm} \mathrm{Hg})$ & $121.4(5.6)$ & $115.8(6.4)$ & $<0.001$ & $123.6(6.0)$ & $133.7(4.1)$ & $<0.001$ & Gaussian \\
$M A P(\mathrm{~mm} \mathrm{Hg})$ & $89.8(3.9)$ & $88.4(4.5)$ & $<0.01$ & $91.6(4.3)$ & $100.0(3.4)$ & $<0.001$ & Gaussian \\
$D A P(\mathrm{~mm} \mathrm{Hg})$ & $70.7(3.5)$ & $71.8(4.0)$ & $<0.01$ & $72.2(3.9)$ & $79.0(3.2)$ & $<0.001$ & Gaussian \\
\hline
\end{tabular}

Values are presented as mean or median (SD or interquartile range - IQR) depending on distribution - Gaussian/non-Gaussian. p-values refer to comparison between neighboring phases - (REST vs. HUT) and (REC vs. MA). Type of data distribution (Gaussian vs. non-Gaussian) is presented in the last column.

\section{Estimation of arterial compliance}

The estimated values of $\tau$ and TPR are presented in Fig. 1. Fig. 1a shows that during stress phases, time constant $\tau$ statistically significantly decreased. In addition, we observed that TPR also statistically significantly increased during both stress phases as it is depicted in Fig. 1b. According to Eq. 1, observed increase in TPR during HUT should lead to a concomitant increase in $\tau$. In contrast, we observed a decrease in $\tau$ during stress phases.

Applying Eq. 3, we were able to estimate values of C. $C$ significantly decreased during both stress phases (Fig. 2a). In Fig. 2b differences in mean $\mathrm{C}$ values between REST-HUT and REC-MA are depicted $\mathrm{C}$ decrease was of similar magnitude during both stress phases.

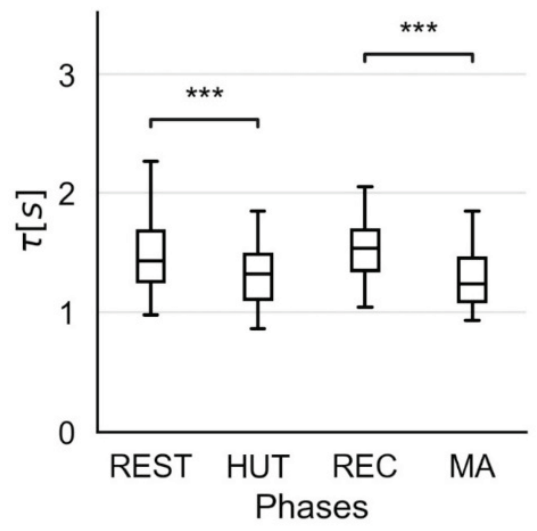

(a) Time constant $T$.

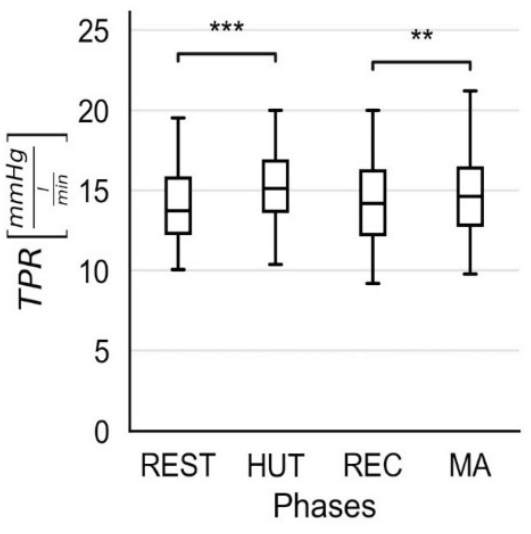

(b) Total peripheral resistence.
Fig. 1. Box plots illustrating changes of $\tau$ (Wilcoxon signed rank test) and TPR (Wilcoxon signed rank test) during four phases of the study protocol. *** indicates a statistically significant difference between stress phase and preceding rest phase. 


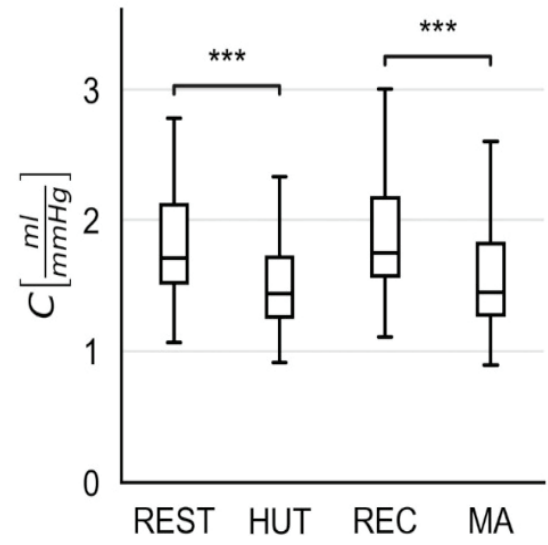

(a) Arterial compliance calculated by Eq. 3 .

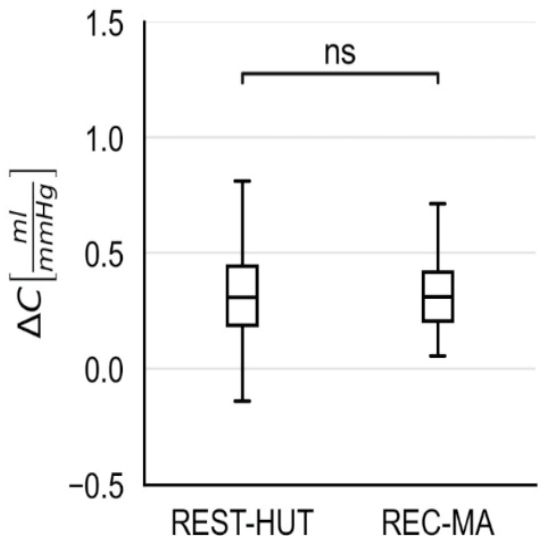

(b) Differences between rest and stress

phases in mean values of $C$.
Fig 2. Box plots illustrating changes of estimated $C$ (Wilcoxon signed rank test) during four phases of the study protocol. *** indicates a statistically significant difference between stress phase and preceding rest phase, ns - not significant.
Relationship between arterial compliance and systolic arterial pressure

An example of the relationships between $\mathrm{C}$ and SAP in one representative subject is illustrated in Fig. 3. Generally, C decreases with an increase in SAP. We observed that the expected relationship between SAP and $\mathrm{C}$ holds for the given phase but it shifts during stress phases (HUT, MA). To minimize the effect of SAP changes during study protocol on $\mathrm{C}$ values, we calculated $C_{120}$ as a value of $\mathrm{C}$ at the SAP of $120 \mathrm{~mm} \mathrm{Hg}$. Using this approach, we found a significant decrease of $\mathrm{C}$ during stress phases. Measured decrease of $\mathrm{C}_{120}$ from REST to HUT phases was more prominent than the decrease from REC to MA phase (Fig. 4b). These differences are reflected by shifts of relationships between $\mathrm{C}$ and SAP during study protocol (Fig. 3).
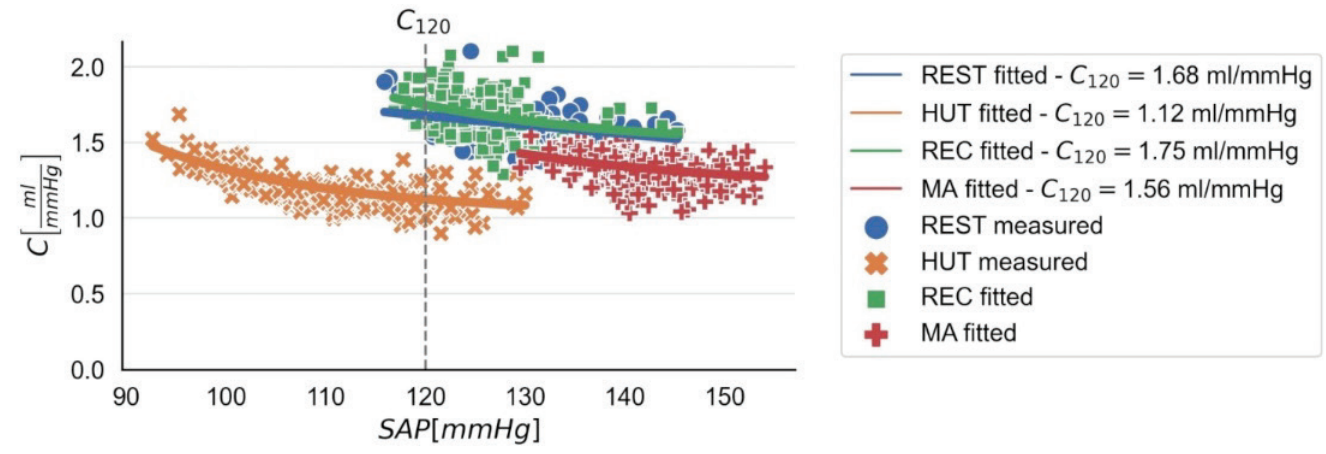

Fig. 3. Relationships between beat-to-beat $C$ and SAP values in one representative subject during all phases with fitted exponential functions and estimated values of $\mathrm{C}_{120}$. With beat-to-beat measurement we obtained large set of data points (C in relation to SAP) with uneven distribution of SAP values potentially affecting the fitted function. To avoid this issue, all data points were equally distributed into 10 bins with random oversampling of recordings.

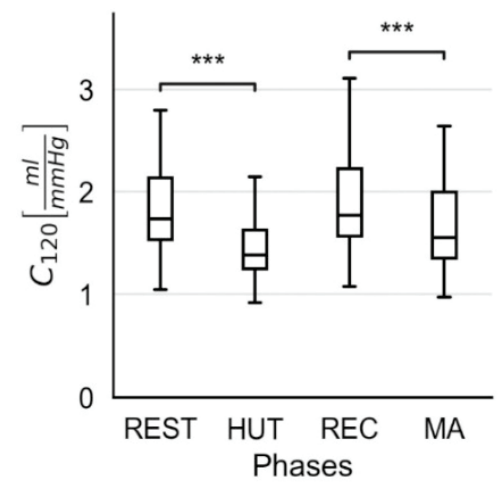

(a) Predicted $\mathrm{C}_{120}$

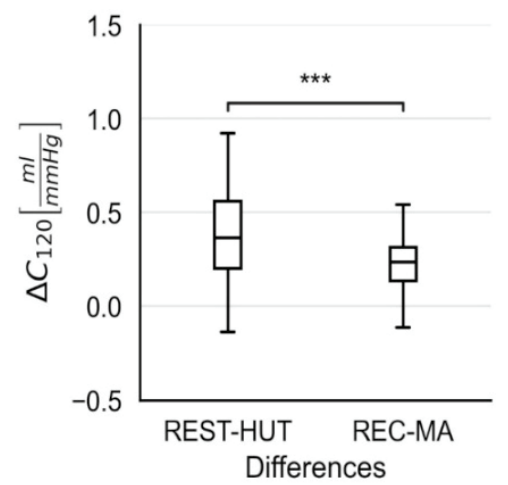

(b) Differences between rest and stress phases in values of $C_{120}$.
Fig. 4. Box plots illustrating changes of predicted values of arterial compliance (Wilcoxon signed rank test) at the systolic arterial pressure of $120 \mathrm{~mm} \mathrm{Hg}$ during four phases of the study protocol. $* * *$ indicates a statistically significant difference between stress phase and preceding rest phase. 


\section{Discussion}

In the present study, we noninvasively assessed changes in $\mathrm{C}$ from rest to stress conditions (orthostasis, mental arithmetics) calculated from diastolic blood pressure decay time constant $\tau$ and TPR changes. We observed a nonlinear relationship between $\mathrm{C}$ and SAP during given physiological state indicating that for the more objective estimation of $\mathrm{C}$ changes in SAP values should be considered. Therefore, an approach estimating a standardized $\mathrm{C}$ value at SAP of $120 \mathrm{~mm} \mathrm{Hg}\left(\mathrm{C}_{120}\right)$ was introduced. The major findings of this study are:

(a) changes in $\tau$ during our study protocol are more attributable to $\mathrm{C}$ than to TPR changes,

(b) the nonlinear relationship between $\mathrm{C}$ and SAP is not fixed but it shifts during stress conditions,

(c) after standardization for SAP changes, $\mathrm{C}$ decreases more during orthostasis than during cognitive challenge,

(d) $\mathrm{C}$ changes are the opposite direction to TPR changes pointing towards the influence of vascular tone on $\mathrm{C}$.

The mechanism of human organism adaptation to orthostatic stress evoked by HUT is well known. During orthostasis, a redistribution of the blood in lower part of the body occurs, thus decreasing cardiac filling and cardiac output with concomitant decrease of blood pressure. In response to these changes, the autonomic nervous system activity changes influence $H R$, sympathetic nerve activity and TPR via baroreflex in order to maintain baseline MAP (Cooper and Hainsworth 2001, Nardone et al. 2018, O'Leary et al. 2003, Sugawara et al. 2012). In addition, a decreased C or increased arterial stiffness were consistently found in previous studies during orthostasis (Hasegawa and Rodbard 1979, Huijben et al. 2012). In contrast to our approach, these studies measured mostly PWV where the effect of SAP fluctuations was not considered potentially introducing confounding factor into $\mathrm{C}$ estimation.

Our raw results of $\mathrm{C}$ estimation without considering an effect of SAP agree with previous findings, that $\mathrm{C}$ decreased during HUT (Fig. 2). In addition to decreased C, TPR and HR increased, whereas SAP and MAP decreased. These changes of hemodynamic parameters are potentially associated with changes of C of varying magnitude (Bank et al. 1995, Cohen et al. 2020, Cox 1975, Czippelova et al. 2019, Hallock and Benson 1937, Tan et al. 2016), therefore, they should be controlled for. Usually it was done by controlling for changes in pressure with an assumption of linear dependency between $\mathrm{C}$ and arterial pressure. However, this is an oversimplification because the relationship between $\mathrm{C}$ and arterial pressure is not linear (Bank et al. 1995, Gavish and Izzo 2016).

To solve these issues, we tried to find relationship between $\mathrm{C}$ and SAP as the first step. We found that $\mathrm{C}$ exponentially decreases with increasing SAP (Fig. 3) which is in agreement with stress-strain relationship (Bank et al. 1995). From this relationship, we were able to control for pressure changes and to compare $\mathrm{C}$ between different phases even when blood pressure changed. SAP during HUT slightly decreased together with a decrease in C. If only SAP effect on C would be considered without a change in arterial properties, a decrease in SAP would be associated with an increase in $\mathrm{C}$. However, the observation of decreased $\mathrm{C}$ indicates that the SAP-C curve shifted (a representative example is presented in Fig. 3) reflecting a change in the arterial tree properties associated with orthostatic reaction. Not surprisingly, we observed after the pressure adjustment that a decrease in $\mathrm{C}$ was even more prominent when normalized to SAP changes using a newly introduced index $C_{120}$. Similar shifts in $C$ were observed in previous studies where an effect of vasomotor tone changes on C was demonstrated (Bank et al. 1995, Pagani et al. 1979). Both studies evoked vasoconstriction or vasodilation using vasoactive substances in adult male subjects (Bank et al.) or adult sheep (Pagani et al.) which led to a shift in relationship between $\mathrm{C}$ or arterial diameter and blood pressure. In contrast to these studies, we were able to measure these shifts in SAP-C curves noninvasively during various physiological states.

In our study, TPR significantly increased during HUT indicating vasoconstriction response - we assume that the observed shift of the relationship between $\mathrm{C}$ and SAP was also associated with vasomotor activity. In agreement with this concept, in previous study a concomitant increase in sympathetic activity to the vessels and arterial stiffness (expressed by PWV) was observed during lower body negative pressure simulating orthostatic challenge without altering arterial blood pressure, HR or TPR indicating independent influence of sympathetic activity on C (Nardone et al. 2018).

Furthermore, Czippelova et al. (2019) observed similar associations between CAVI (arterial stiffness index independent of pressure changes (Hayashi et al. 2015)) and vascular resistance. They observed that the young obese subjects have lower CAVI indicating higher 
C, together with a decreased TPR. Moreover, they found lower cardiovascular sympathetic activity in this group. These results also point towards the influence of sympathetic activity on changes of $\mathrm{C}$.

Mental stress elicits sympathetic activity increase causing an increase in blood pressure, HR and vasoconstriction in the renal and splanchnic vascular beds accompanied by the vasodilation in skeletal muscles (Kuipers et al. 2008, Lindvall et al. 1991, Wasmund et al. 2002). Furthermore, MA was found to decrease $C$ as well demonstrated indirectly using PWV, CAVI and augmentation index measurement (Lydakis et al. 2008, Matsumura et al. 2019, Vlachopoulos et al. 2006). In our study, MA task evoked similar reactions - a significant increase in blood pressure measures, HR (Table 1) and TPR (Fig. 1b), whereas C significantly decreased (Fig. 2a). Knowing previously mentioned potential association of SAP with $\mathrm{C}$, we controlled for pressure via fitted exponential function using $\mathrm{C}_{120}$ index (Fig. 3) and we still observed decreased C (Fig. 4a). However, this decrease was smaller compared to orthostasis potentially corresponding to the smaller increase in TPR during MA compared to HUT.

Similar to $\mathrm{C}_{120}$ index, $\beta$ stiffness index and CAVI are considered pressure independent indices of arterial stiffness. However, we believe that proposed $\mathrm{C}_{120}$ index might be a valuable alternative to the established indexes having in mind differences in applicability and limitations of each index. $\beta$ stiffness index quantifies only local arterial stiffness. Non-invasive and beat-to-beat estimation of its value requires simultaneous recording of changes in cross-sectional area of the selected artery and corresponding changes in blood pressure introducing inevitable methodologic difficulties (Hayashi et al. 1980). On the other hand, CAVI quantifies systemic arterial stiffness because it is measured on the much larger segment of arterial tree between heart and ankle. However, beat-to-beat estimation of CAVI is currently not possible. Furthermore, recent studies challenged the ability of CAVI to quantify pressure independent arterial stiffness. New index $\mathrm{CAVI}_{0}$ solves this problem (Giudici et al. 2021). Another disadvantage of CAVI or $\mathrm{CAVI}_{0}$ index is a need to calculate PWV. To accurately calculate PWV it is required to know the distance within arterial system and the time needed for propagation of pulse wave from one measurement point to another. The main problem is with an estimation of distance - it is usually estimated from the body height of the measured subject potentially introducing an error. For CAVI index calculation, PWV is squared which even more increases this error.

$\mathrm{C}_{120}$ index is derived from beat-to-beat estimation of systemic arterial compliance and blood pressure (SAP), rendering it an only index directly derived from a relation between $\mathrm{C}$ and SAP. To be able to estimate $\mathrm{C}$ with proposed method it is required to record continuous peripheral blood pressure curve and SV for every beat. Both these signals are easily measured by volume-clamp method and impedance cardiography. Furthermore, beat-to-beat values of $\mathrm{C}$ measured by proposed method might be a valuable tool for the analysis of causal relationships between cardiovascular measures. The most important disadvantage of $\mathrm{C}_{120}$ index is in its requirement of longer measurement than other indexes for proper estimation of the relation between $\mathrm{C}$ and SAP.

With the noninvasiveness of our approach, measurement errors could be introduced in the estimation of cardiovascular parameters. Furthermore, the method of time constant $\tau$ estimation was validated only on animal model (Arai et al. 2008). In order to estimate values of C, the Windkessel model was used which oversimplifies the arterial tree, e.g. the arterial tree is modeled with separate "conduit" (TPR) and "cushioning" (C) elements (Eq. 1) or the pulse wave velocity is considered as infinitely high (Laurent et al. 2006). Moreover, C could be associated with several other factors (e.g. heart rate). In this study we only controlled for a well-documented effect of arterial blood pressure changes. We did not measure sympathetic nerve activity to the vessels directly because of its invasiveness.

From the clinical point of view, our results demonstrate that $\mathrm{C}$ estimation should consider arterial blood pressure perturbations. In addition, changes in $\mathrm{C}$ associated with short- or long-term vascular tone perturbations should be considered when interpreting $\mathrm{C}$ as an index of structural vascular changes (e.g. associated with atherosclerosis). Because of the observed short-term effects of physiological state changes on $\mathrm{C}$ values, our results also indicate an importance of measurement conditions standardization during examination of vascular stiffness (or surrogate indices).

We conclude that we introduced a Windkessel model-based method for noninvasive assessment of beat to beat $\mathrm{C}$ changes in the context of instantaneous arterial blood pressure changes. In relatively large group of young and healthy volunteers we demonstrated that $\mathrm{C}$ changes also on a short time scale independent on blood pressure changes it decreases during cognitive load and even more 
prominently during orthostatic test. These changes potentially reflect changes in vasomotor tone directed to arterial tree.

\section{Acknowledgements}

The study was supported by grants VEGA 1/0199/19, 1/0200/19, 1/0283/21 and Grant UK/140/2021.

\section{Conflict of Interest}

There is no conflict of interest.

\section{References}

ARAI T, LEE K, COHEN RJ: A novel algorithm to continuously monitor change of total peripheral resistance using peripheral arterial blood pressure values for prediction of orthostatic intolerance. In: Proceedings of the International Astronautical Congress Glasgow. Scotland, p IAC-08.A1.2.13, 2008.

ARAI T, LEE K, STENGER MB, PLATTS SH, MECK JV, COHEN RJ: Preliminary application of a novel algorithm to monitor changes in pre-flight total peripheral resistance for prediction of post-flight orthostatic intolerance in astronauts. Acta Astronaut 68: 770-777, 2011. https://doi.org/10.1016/j.actaastro.2010.10.008

BANK AJ, WILSON RF, KUBO SH, HOLTE JE, DRESING TJ, WANG H: Direct effects of smooth muscle relaxation and contraction on in vivo human brachial artery elastic properties. Circ Res 77: 1008-1016, 1995. https://doi.org/10.1161/01.RES.77.5.1008

CHEMLA D, ANTONY I, LECARPENTIER Y, NITENBERG A: Contribution of systemic vascular resistance and total arterial compliance to effective arterial elastance in humans. Am J Physiol Heart Circ Physiol 285: H614-H620, 2003. https://doi.org/10.1152/ajpheart.00823.2002

COHEN J, PIGNANELLI C, BURR J: The effect of body position on measures of arterial stiffness in humans. J Vasc Res 57: 143-151, 2020. https://doi.org/10.1159/000506351

COOPER VL, HAINSWORTH R: Carotid baroreceptor reflexes in humans during orthostatic stress. Exp Physiol 86: 677-681, 2001. https://doi.org/10.1113/eph8602213

COX RH: Arterial wall mechanics and composition and the effects of smooth muscle activation. Am J Physiol 229: 807-812, 1975. https://doi.org/10.1152/ajplegacy.1975.229.3.807

CZIPPELOVA B, TURIANIKOVA Z, KROHOVA J, WISZT R, LAZAROVA Z, POZORCIAKOVA K, CILJAKOVA M, JAVORKA M: Arterial stiffness and endothelial function in young obese patients - vascular resistance matters. J Atheroscler Thromb 26: 1015-1025, 2019. https://doi.org/10.5551/jat.47530

GAVISH B, IZZO JL JR: Arterial stiffness: Going a step beyond. Am J Hypertens 29: 1223-1233, 2016. https://doi.org/10.1093/ajh/hpw061

GIUDICI A, KHIR AW, REESINK KD, DELHAAS T, SPRONCK B: Five years of cardio-ankle vascular index (CAVI) and CAVI0: how close are we to a pressure-independent index of arterial stiffness? J Hypertens 39 : 2128-2138, 2021. https://doi.org/10.1097/HJH.0000000000002928

HALLOCK P, BENSON IC: Studies on the elastic properties of human isolated aorta. J Clin Invest 16: 595-602, 1937. https://doi.org/10.1172/JCI100886

HASEGAWA M, RODBARD S: Effect of posture on arterial pressures, timing of the arterial sounds and pulse wave velocities in the extremities. Cardiology 64: 122-132, 1979. https://doi.org/10.1159/000170585

HAYASHI K, HANDA H, NAGASAWA S, OKUMURA A, MORITAKE K: Stiffness and elastic behavior of human intracranial and extracranial arteries. J Biomech 13: 175-184, 1980. https://doi.org/10.1016/0021-9290(80)90191-8

HAYASHI K, YAMAMOTO T, TAKAHARA A, SHIRAI K: Clinical assessment of arterial stiffness with cardio-ankle vascular index: theory and applications. J Hypertens 33: 1742-1757; discussion 1757, 2015. https://doi.org/10.1097/HJH.0000000000000651

HUIJBEN AMT, MATTACE-RASO FUS, DEINUM J, LENDERS J, VAN DEN MEIRACKER AH: Aortic augmentation index and pulse wave velocity in response to head-up tilting: effect of autonomic failure. J Hypertens 30 : 307-314, 2012. https://doi.org/10.1097/HJH.0b013e32834f09ee 
KROEKER EJ, WOOD EH: Comparison of simultaneously recorded central and peripheral arterial pressure pulses during rest, exercise and tilted position in man. Circ Res 3: 623-632, 1955. https://doi.org/10.1161/01.RES.3.6.623

KUIPERS NT, SAUDER CL, CARTER JR, RAY CA: Neurovascular responses to mental stress in the supine and $\begin{array}{lllllll}\text { upright } & \text { postures. } & \mathrm{J} & \text { Appl } & \text { Physiol } & \text { (1985) } & \text { 104: }\end{array}$ https://doi.org/10.1152/japplphysiol.01285.2007

LANTELME P, MESTRE C, LIEVRE M, GRESSARD A, MILON H: Heart rate: an important confounder of pulse $\begin{array}{lllll}\text { wave } & \text { velocity } & \text { assessment. } & \text { Hypertension }\end{array}$ https://doi.org/10.1161/01.HYP.0000019132.41066.95

LAURENT S, COCKCROFT J, VAN BORTEL L, BOUTOUYRIE P, GIANNATTASIO C, HAYOZ D, PANNIER B, VLACHOPOULOS C, WILKINSON I, STRUIJKER-BOUDIER H, EUROPEAN NETWORK FOR NON-INVASIVE INVESTIGATION OF LARGE ARTERIES: Expert consensus document on arterial stiffness: methodological issues and clinical applications. Eur Heart J 27: 2588-2605, 2006. https://doi.org/10.1093/eurheartj/ehl254

LEVENSON JA, SAFAR ME, SIMON AC, KHEDER AI, DAOU JN, LEVY BI: Systemic arterial compliance and diastolic runoff in essential hypertension. Angiology 32: 402-413, 1981. https://doi.org/10.1177/000331978103200606

LINDVALL K, KAHAN T, DE U, ÖSTERGREN J, HJEMDAHL P: Stress-induced changes in blood pressure and left ventricular function in mild hypertension. Clin Cardiol 14: 125-132, 1991. https://doi.org/10.1002/clc.4960140208

LYDAKIS C, MOMEN A, BLAHA C, GUGOFF S, GRAY K, HERR M, LEUENBERGER UA, SINOWAY LI: Changes of central haemodynamic parameters during mental stress and acute bouts of static and dynamic exercise. J Hum Hypertens 22: 320-328, 2008. https://doi.org/10.1038/jhh.2008.4

MATSUMURA K, NOGUCHI H, ROLFE P, YAMAKOSHI T, MATSUOKA Y: Differential effect of two mental stress tasks on arterial stiffness. Jpn Psychol Res 61: 249-261, 2019. https://doi.org/10.1111/jpr.12235

MCENIERY CM, YASMIN, HALL IR, QASEM A, WILKINSON IB, COCKCROFT JR: Normal vascular aging: Differential effects on wave reflection and aortic pulse wave velocity: The Anglo-Cardiff Collaborative Trial (ACCT). J Am Coll Cardiol 46: 1753-1760, 2005. https://doi.org/10.1016/j.jacc.2005.07.037

NAGAI Y, HELWEGEN J, FLEG JL, BEEMER MK, EARLEY CJ, METTER EJ: Associations of aortic Windkessel function with age, gender and cardiovascular risk factors. Ultrasound Med Biol 27: 1207-1210, 2001. https://doi.org/10.1016/S0301-5629(01)00445-8

NARDONE M, INCOGNITO ANTHONY V, MILLAR PHILIP J: Evidence for pressure-independent sympathetic modulation of central pulse wave velocity. J Am Heart Assoc 7: e007971, 2018. https://doi.org/10.1161/JAHA.117.007971

O'LEARY DD, KIMMERLY DS, CECHETTO AD, SHOEMAKER JK: Differential effect of head-up tilt on cardiovagal and sympathetic baroreflex sensitivity in humans. Exp Physiol 88: 769-774, 2003. https://doi.org/10.1113/eph8802632

O'ROURKE MF, HASHIMOTO J: Mechanical factors in arterial aging: A clinical perspective. J Am Coll Cardiol 50: 1-13, 2007. https://doi.org/10.1016/j.jacc.2006.12.050

PAGANI M, MIRSKY I, BAIG H, MANDERS WT, KERKHOF P, VATNER SF: Effects of age on aortic pressurediameter and elastic stiffness-stress relationships in unanesthetized sheep. Circ Res 44: 420-429, 1979. https://doi.org/10.1161/01.RES.44.3.420

SAGAWA K, LIE RK, SCHAEFER J: Translation of Otto Frank's paper "Die Grundform des Arteriellen Pulses" Zeitschrift für Biologie 37: 483-526 (1899). J Mol Cell Cardiol 22: 253-254, 1990. https://doi.org/10.1016/0022-2828(90)91459-K

SCHROEDER EC, ROSENBERG AJ, HILGENKAMP TIM, WHITE DW, BAYNARD T, FERNHALL B: Effect of upper body position on arterial stiffness: influence of hydrostatic pressure and autonomic function. J Hypertens 35: 2454-2461, 2017. https://doi.org/10.1097/HJH.0000000000001481

STERGIOPULOS N, MEISTER JJ, WESTERHOF N: Evaluation of methods for estimation of total arterial compliance. Am J Physiol 268: H1540-H1548, 1995. https://doi.org/10.1152/ajpheart.1995.268.4.H1540 
SUGAWARA J, WILLIE CK, MIYAZAWA T, KOMINE H, AINSLE PN, OGOH S: Effects of transient change in carotid arterial stiffness on arterial baroreflex during mild orthostatic stimulation. Artery Res 6: 130-135, 2012. https://doi.org/10.1016/j.artres.2012.05.002

TAN I, SPRONCK B, KIAT H, BARIN E, REESINK KD, DELHAAS T, AVOLIO AP, BUTLIN M: Heart rate dependency of large artery stiffness. Hypertension 68: 236-242, 2016. https://doi.org/10.1161/HYPERTENSIONAHA.116.07462

VLACHOPOULOS C, KOSMOPOULOU F, ALEXOPOULOS N, IOAKEIMIDIS N, SIASOS G, STEFANADIS C: Acute mental stress has a prolonged unfavorable effect on arterial stiffness and wave reflections. Psychosom Med 68: 231-237, 2006. https://doi.org/10.1097/01.psy.0000203171.33348.72

WASMUND WL, WESTERHOLM EC, WATENPAUGH DE, WASMUND SL, SMITH ML: Interactive effects of mental and physical stress on cardiovascular control. J Appl Physiol (1985) 92: 1828-1834, 2002. https://doi.org/10.1152/japplphysiol.00019.2001 